\title{
EVALUATING PRIME POWER GAUSS AND JACOBI SUMS
}

\author{
MISTY OSTERGAARD, VINCENT PIGNO AND CHRISTOPHER PINNER
}

Abstract. We show that for any $\bmod p^{m}$ characters, $\chi_{1}, \ldots, \chi_{k}$, with at least one $\chi_{i}$ primitive $\bmod p^{m}$, the Jacobi sum,

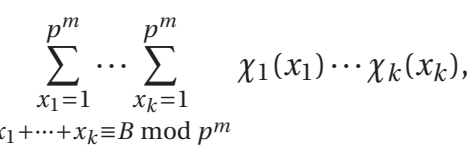

has a simple evaluation when $m$ is sufficiently large (for $m \geq 2$ if $p \nmid B$ ). As part of the proof we give a simple evaluation of the $\bmod p^{m}$ Gauss sums when $m \geq 2$ that differs slightly from existing evaluations when $p=2$.

\section{Introduction}

For multiplicative characters $\chi_{1}$ and $\chi_{2} \bmod q$ one defines the classical Jacobi sum by

$$
J\left(\chi_{1}, \chi_{2}, q\right):=\sum_{x=1}^{q} \chi_{1}(x) \chi_{2}(1-x) .
$$

More generally for $k$ characters $\chi_{1}, \ldots, \chi_{k} \bmod q$ one can define

$$
J\left(\chi_{1}, \ldots, \chi_{k}, q\right)=\sum_{\substack{x_{1}=1 \\ x_{1}+\cdots+x_{k} \equiv 1 \bmod q}}^{q} \ldots \sum_{\substack{x_{k}=1\\}}^{q} \chi_{1}\left(x_{1}\right) \cdots \chi_{k}\left(x_{k}\right) .
$$

If the $\chi_{i}$ are mod $r s$ characters with $(r, s)=1$, then, writing $\chi_{i}=\chi_{i}^{\prime} \chi_{i}^{\prime \prime}$ where $\chi_{i}^{\prime}$ and $\chi_{i}^{\prime \prime}$ are mod $r$ and $\bmod s$ characters respectively, it is readily seen (e.g. [13, Lemma 2]) that

$$
J\left(\chi_{1}, \ldots, \chi_{k}, r s\right)=J\left(\chi_{1}^{\prime}, \ldots, \chi_{k}^{\prime}, r\right) J\left(\chi_{1}^{\prime \prime}, \ldots, \chi_{k}^{\prime \prime}, s\right)
$$

Hence, one usually only considers the case of prime power moduli $q=p^{m}$.

Received April 27, 2016, accepted October 19, 2016.

2010 Mathematics Subject Classification. Primary: 11L05; Secondary: 11L03, 11L10.

Key words and phrases. Gauss sums, Jacobi sums, character sums, exponential sums.

Corresponding author: Vincent Pigno. 
Zhang \& Yao [12] showed that the sums (1) can in fact be evaluated explicitly when $m$ is even (and $\chi_{1}, \chi_{2}$ and $\chi_{1} \chi_{2}$ are primitive $\bmod p^{m}$ ). Working with a slightly more general binomial character sum two of the authors [9] showed that techniques of Cochrane \& Zheng [3] (see also [2]) can be used to obtain an evaluation of (1) for any $m>1$ with $p$ an odd prime. Zhang \& Xu [13] considered the general case, (2), and assuming that $\chi, \chi^{n_{1}}, \ldots, \chi^{n_{k}}$, and $\chi^{n_{1}+\cdots+n_{k}}$ are primitive characters modulo $p^{m}$, obtained

$$
J\left(\chi^{n_{1}}, \ldots, \chi^{n_{k}}, p^{m}\right)=p^{\frac{1}{2}(k-1) m} \bar{\chi}\left(u^{u}\right) \chi\left(n_{1}^{n_{1}} \ldots n_{k}^{n_{k}}\right), u:=n_{1}+\cdots+n_{k},
$$

when $m$ is even, and

$$
J\left(\chi^{n_{1}}, \ldots, \chi^{n_{k}}, p^{m}\right)=p^{\frac{1}{2}(k-1) m} \bar{\chi}\left(u^{u}\right) \chi\left(n_{1}^{n_{1}} \ldots n_{k-1}^{n_{k-1}}\right) \begin{cases}\varepsilon_{p}^{k-1}\left(\frac{u n_{1} \ldots n_{k}}{p}\right), & \text { if } p \neq 2 \\ \left(\frac{2}{u n_{1} \ldots n_{k}}\right) & \text { if } p=2\end{cases}
$$

when $m, k, n_{1}, \ldots, n_{k}$ are all odd, where $\left(\frac{m}{n}\right)$ is the Jacobi symbol and (defined more generally for later use)

$$
\varepsilon_{p^{m}}:= \begin{cases}1, & \text { if } p^{m} \equiv 1 \bmod 4, \\ i, & \text { if } p^{m} \equiv 3 \bmod 4 .\end{cases}
$$

In this paper we give an evaluation for all $m>1$ (i.e. irrespective of the parity of $k$ and the $n_{i}$ ). In fact we evaluate the slightly more general sum

$$
J_{B}\left(\chi_{1}, \ldots, \chi_{k}, p^{m}\right)=\sum_{\substack{x_{1}=1 \\ x_{1}+\cdots+x_{k} \equiv B \bmod p^{m}}}^{p^{m}} \chi_{x_{k}=1}^{p^{m}} \chi_{1}\left(x_{1}\right) \cdots \chi_{k}\left(x_{k}\right) .
$$

Of course when $B=p^{n} B^{\prime}, p \nmid B^{\prime}$ the simple change of variables $x_{i} \mapsto B^{\prime} x_{i}$ gives

$$
J_{B}\left(\chi_{1}, \ldots, \chi_{k}, p^{m}\right)=\chi_{1} \cdots \chi_{k}\left(B^{\prime}\right) J_{p^{n}}\left(\chi_{1}, \ldots, \chi_{k}, p^{m}\right) .
$$

For example, $J_{B}\left(\chi_{1}, \ldots, \chi_{k}, p^{m}\right)=\chi_{1} \cdots \chi_{k}(B) J\left(\chi_{1}, \ldots, \chi_{k}, p^{m}\right)$ when $p \nmid B$. From the change of variables $x_{i} \mapsto-x_{k} x_{i}, 1 \leq i<k$ one also sees that

$$
J_{p^{m}}\left(\chi_{1}, \ldots, \chi_{k}, p^{m}\right)= \begin{cases}\phi\left(p^{m}\right) \chi_{k}(-1) J\left(\chi_{1}, \ldots, \chi_{k-1}, p^{m}\right), & \text { if } \chi_{1} \cdots \chi_{k}=\chi_{0}, \\ 0, & \text { if } \chi_{1} \cdots \chi_{k} \neq \chi_{0},\end{cases}
$$

where $\chi_{0}$ denotes the principal character, so we assume that $B=p^{n}$ with $n<m$.

For $p$ odd let $a$ be a primitive root $\bmod p^{s}$ for all $s$. We define the integer $r$ by

$$
a^{\phi(p)}=1+r p, \quad p \nmid r .
$$

For a character $\chi_{i} \bmod p^{m}$ we define the integer $c_{i}$ by

$$
\chi_{i}(a)=e_{\phi\left(p^{m}\right)}\left(c_{i}\right), \quad 1 \leq c_{i} \leq \phi\left(p^{m}\right) .
$$


Note, $p \nmid c_{i}$ exactly when $\chi_{i}$ is primitive. For $p=2, m=2$ we take $a=-1$ in (7).

For $p=2$ and $m \geq 3$ we need two generators -1 and 5 for $\mathbb{Z}_{2^{m}}^{*}$ and define $c_{i}$ by

$$
\chi_{i}(5)=e_{2^{m-2}}\left(c_{i}\right), \quad 1 \leq c_{i} \leq 2^{m-2},
$$

with $\chi_{i}$ primitive exactly when $2 \nmid c_{i}$.

Theorem 1.1. Let $p$ be a prime and $m \geq n+2$. Suppose that $\chi_{1}, \ldots, \chi_{k}$, are $k \geq 2$ characters mod $p^{m}$ with at least one of them primitive.

If $\chi_{1}, \ldots, \chi_{k}$ are not all primitive $\bmod p^{m}$ or $\chi_{1} \ldots \chi_{k}$ is not induced by a primitive mod $p^{m-n}$ character, then $J_{p^{n}}\left(\chi_{1}, \ldots, \chi_{k}, p^{m}\right)=0$.

If $\chi_{1}, \ldots, \chi_{k}$ are primitive $\bmod p^{m}$ and $\chi_{1} \cdots \chi_{k}$ is primitive $\bmod p^{m-n}$, then

$$
J_{p^{n}}\left(\chi_{1}, \ldots, \chi_{k}, p^{m}\right)=p^{\frac{1}{2}(m(k-1)+n)} \frac{\chi_{1}\left(c_{1}\right) \cdots \chi_{k}\left(c_{k}\right)}{\chi_{1} \cdots \chi_{k}(v)} \delta
$$

where for $p$ odd

$$
\delta=\left(\frac{-2 r}{p}\right)^{m(k-1)+n}\left(\frac{v}{p}\right)^{m-n}\left(\frac{c_{1} \cdots c_{k}}{p}\right)^{m} \varepsilon_{p^{m}}^{k} \varepsilon_{p^{m-n}}^{-1},
$$

with an extra factor $e^{2 \pi i r v / 3}$ needed when $p=m-n=3, n>0$, and for $p=2$ and $m-n \geq 5$,

$$
\delta=\left(\frac{2}{v}\right)^{m-n}\left(\frac{2}{c_{1} \cdots c_{k}}\right)^{m} \omega^{\left(2^{n}-1\right) v},
$$

with $\varepsilon_{p^{m}}$ as defined in (5), the r and $c_{i}$ as in (6) and (7) or (8), and

$$
v:=p^{-n}\left(c_{1}+\cdots+c_{k}\right), \quad \omega:=e^{\pi i / 4} .
$$

For $m \geq 5$ and $m-n=2,3$ or 4 the formula (10) for $\delta$ should be multiplied by $\omega, \omega^{1+\chi_{1} \cdots \chi_{k}(-1)}$, or $\chi_{1} \cdots \chi_{k}(-1) \omega^{2 v}$ respectively.

Of course it is natural to assume that at least one of the $\chi_{1}, \ldots, \chi_{k}$ is primitive, otherwise we can reduce the sum to a $\bmod p^{m-1}$ sum. For $n=0$ and $\chi_{1}, \ldots, \chi_{k}$, and $\chi_{1} \cdots \chi_{k}$ all primitive $\bmod p^{m}$, our result simplifies to

$$
J\left(\chi_{1}, \ldots, \chi_{k}, p^{m}\right)=p^{\frac{m(k-1)}{2}} \frac{\chi_{1}\left(c_{1}\right) \cdots \chi_{k}\left(c_{k}\right)}{\chi_{1} \cdots \chi_{k}(v)} \delta, \quad v=c_{1}+\cdots+c_{k},
$$

with

$$
\delta= \begin{cases}1, & \text { if } m \text { is even, } \\ \left(\frac{v c_{1} \cdots c_{k}}{p}\right)\left(\frac{-2 r}{p}\right)^{k-1} \varepsilon_{p}^{k-1}, & \text { if } m \text { is odd and } p \neq 2, \\ \left(\frac{2}{v c_{1} \cdots c_{k}}\right), & \text { if } m \geq 5 \text { is odd and } p=2 .\end{cases}
$$


In the remaining $n=0$ case, $p=2, m=3$ we have $J\left(\chi_{1}, \ldots, \chi_{k}, 2^{3}\right)=2^{\frac{3}{2}(k-1)}(-1)^{\left\lfloor\frac{\ell}{2}\right\rfloor}$ where $\ell$ denotes the number of characters $1 \leq i \leq k$ with $\chi_{i}(-1)=-1$.

When the $\chi_{i}=\chi^{n_{i}}$ for some primitive $\bmod p^{m}$ character $\chi$, we can write $c_{i}=n_{i} c$ (where $c$ is determined by $\chi(a)$ as in (7) or (8)), and for $m$ even we recover the form (3), and for $m$ odd we recover (4) but with the addition of a factor $\left(\frac{-2 r c}{p}\right)^{k-1}$ for $p \neq 2$, which of course can be ignored when $k$ is odd as assumed in [13].

For completeness we observe that in the few remaining $m \geq n+2$ cases, (9) becomes

$$
J_{p^{n}}\left(\chi_{1}, \ldots, \chi_{k}, p^{m}\right)=2^{\frac{1}{2}(m(k-1)+n)} \begin{cases}-i \omega^{k-\sum_{i=1}^{k} \chi_{i}(-1)}, & \text { if } m=3, n=1 \\ \omega^{\chi_{1} \cdots \chi_{k}(-1)-1-v} \prod_{i=1}^{k} \chi_{i}\left(-c_{i}\right), & \text { if } m=4, n=1 \\ i^{1-v} \prod_{i=1}^{k} \chi_{i}\left(c_{i}\right), & \text { if } m=4, n=2 .\end{cases}
$$

Our proof of Theorem 1.1 involves expressing the Jacobi sum (2) in terms of classical Gauss sums

$$
G\left(\chi, p^{m}\right):=\sum_{x=1}^{p^{m}} \chi(x) e_{p^{m}}(x),
$$

where $\chi$ is a $\bmod p^{m}$ character and $e_{y}(x):=e^{2 \pi i x / y}$. Writing (1) in terms of Gauss sums is well known for the mod $p$ sums and the corresponding result for (2) can be found, along with many other properties of Jacobi sums, in Berndt, Evans and Williams [1, Theorem 2.1.3 \& Theorem 10.3.1 ] or Lidl and Niederreiter [5, Theorem 5.21]. There the results are stated for sums over finite fields, $\mathbb{F}_{p^{m}}$, so it is not surprising that such expressions exist in the less studied mod $p^{m}$ case. When $\chi_{1}, \ldots, \chi_{k}$, and $\chi_{1} \cdots \chi_{k}$ are primitive, Zhang \& Yao [12, Lemma 3] for $k=2$, and Zhang and $\mathrm{Xu}[13$, Lemma 1] for general $k$, showed that

$$
J\left(\chi_{1}, \ldots, \chi_{k}, p^{m}\right)=\frac{\prod_{i=1}^{k} G\left(\chi_{i}, p^{m}\right)}{G\left(\chi_{1} \ldots \chi_{k}, p^{m}\right)} .
$$

In Theorem 2.2 we obtain a similar expansion for $J_{p^{n}}\left(\chi_{1}, \ldots, \chi_{k}, p^{m}\right)$. Wang [11, Theorem 2.5] had in fact already obtained such an expression for Jacobi sums over much more general rings of residues modulo prime powers. (However, we use a slightly different form to avoid splitting into cases as there.) As we show in Theorem 2.1, the mod $p^{m}$ Gauss sums can be evaluated explicitly using the method of Cochrane and Zheng [3] when $m \geq 2$.

For $m=n+1$ and at least one $\chi_{i}$ primitive, the Jacobi sum is still zero unless all the $\chi_{i}$ are primitive $\bmod p^{m}$ and $\chi_{1} \cdots \chi_{k}$ is a mod $p$ character. Then we can say that $\left|J_{p^{n}}\left(\chi_{1}, \ldots, \chi_{k}, p^{m}\right)\right|$ $=p^{\frac{1}{2} m k-1}$ if $\chi_{1} \cdots \chi_{k}=\chi_{0}$ and $p^{\frac{1}{2}(m k-1)}$ otherwise, but an explicit evaluation in the latter case is equivalent to an explicit evaluation of the mod $p$ Gauss sum $G\left(\chi_{1} \cdots \chi_{k}, p\right)$ when $m \geq 2$. 


\section{Gauss sums}

In order to use the result from [4] we must establish some congruence relationships. For $p$ odd let $a$ be a primitive root $\bmod p^{m}, m \geq 2$. We define the integers $R_{j}, j \geq 1$, by

$$
a^{\phi\left(p^{j}\right)}=1+R_{j} p^{j}
$$

Note that for $j \geq i$,

$$
R_{j} \equiv R_{i} \bmod p^{i}
$$

For $p=2$ and $m \geq 3$ we define the integers $R_{j}, j \geq 2$, by

$$
5^{2^{j-2}}=1+R_{j} 2^{j} .
$$

Noting that $R_{i}^{2} \equiv 1 \bmod 8$, we get

$$
R_{i+1}=R_{i}+2^{i-1} R_{i}^{2} \equiv R_{i}+2^{i-1} \bmod 2^{i+2} .
$$

For $j \geq i+2$ this gives the relationships,

$$
R_{j} \equiv R_{i+2} \equiv R_{i+1}+2^{i} \equiv\left(R_{i}+2^{i-1}\right)+2^{i} \equiv R_{i}-2^{i-1} \bmod 2^{i+1}
$$

and

$$
R_{j} \equiv\left(R_{i-1}+2^{i-2}\right)-2^{i-1} \equiv R_{i-1}-2^{i-2} \bmod 2^{i+1} .
$$

We shall need an explicit evaluation of the $\bmod p^{m}, m \geq 2$, Gauss sums. The form we use comes from applying the technique of Cochrane \& Zheng [3] as formulated in [8]. For $p$ odd this is essentially the same as Cochrane \& Zheng $[4, \$ 10]$ but here we use the simpler $R_{j}$ as opposed to the $p$-adic logarithm used in [4]; an adjustment to their formula is also needed in the case $p^{m}=3^{3}$ (see errata for [3]). For $p=2$ we use the same technique to get a new evaluation of the Gauss sum. Variations can be found in Odoni [7] and Mauclaire [6] (see also Berndt \& Evans [1, §1.6 ] and Cochrane [2, Theorem 6.1]).

Theorem 2.1. Suppose that $\chi$ is a mod $p^{m}$ character with $m \geq 2$. If $\chi$ is imprimitive, then $G\left(\chi, p^{m}\right)=0$. If $\chi$ is primitive, then

$$
G\left(\chi, p^{m}\right)=p^{\frac{m}{2}} \chi\left(-c R_{j}^{-1}\right) e_{p^{m}}\left(-c R_{j}^{-1}\right) \begin{cases}\left(\frac{-2 r c}{p}\right)^{m} \varepsilon_{p^{m}}, & \text { if } p \neq 2, p^{m} \neq 27, \\ \left(\frac{2}{c}\right)^{m} \omega^{c}, & \text { if } p=2 \text { and } m \geq 5,\end{cases}
$$

for any $j \geq\left\lceil\frac{m}{2}\right\rceil$ when $p$ is odd and any $j \geq\left\lceil\frac{m}{2}\right\rceil+2$ when $p=2$.

When $p^{m}=27$ an extra factor $e_{3}(-r c)$ is needed. For the remaining cases

$$
G\left(\chi, 2^{m}\right)=2^{\frac{m}{2}} \begin{cases}i, & \text { if } m=2, \\ \omega^{1-\chi(-1)}, & \text { if } m=3 \\ \chi(-c) e_{16}(-c), & \text { if } m=4\end{cases}
$$


Here $x^{-1}$ denotes the inverse of $x \bmod p^{m}$, and $r, c$ and $R_{j}$ are as in (6), (7) or (8), and (14) or (16), $\omega$ as in (11), and $\varepsilon_{p^{m}}$ as in (5).

Proof. When $p$ is odd, $p^{m} \neq 27$, [8, Theorem 2.1] gives

$$
G\left(\chi, p^{m}\right)=p^{m / 2} \chi(\alpha) e_{p^{m}}(\alpha)\left(\frac{-2 r c}{p^{m}}\right) \varepsilon_{p^{m}}
$$

where $\alpha$ is a solution of

$$
c+R_{J} x \equiv 0 \bmod p^{J}, \quad J:=\left\lceil\frac{m}{2}\right\rceil,
$$

and $G\left(\chi, p^{m}\right)=0$ if no solution exists. So, if $p \mid c$, there is no solution and $G\left(\chi, p^{m}\right)=0$. If, however, $p \nmid c$, by (15) we may take $\alpha=-c R_{J}^{-1} \equiv-c R_{j}^{-1} \bmod p^{J}$ for any $j \geq J$. When $p^{m}=27$ we need the extra factor $e_{3}(-r c)$.

If $p=2, m \geq 6$, and $\chi$ is primitive, then [8, Theorem 5.1] gives

$$
G\left(\chi, 2^{m}\right)=2^{m / 2} \chi(\alpha) e_{2^{m}}(\alpha) \begin{cases}1, & \text { if } m \text { is even, } \\ \frac{1+(-1)^{\lambda} i^{R} J^{c}}{\sqrt{2}}, & \text { if } m \text { is odd }\end{cases}
$$

where $\alpha$ is a solution to

$$
c+R_{J} x \equiv 0 \bmod 2^{\left\lfloor\frac{m}{2}\right\rfloor},
$$

and $c+R_{J} \alpha=2^{\left\lfloor\frac{m}{2}\right\rfloor} \lambda$. If $\chi$ is imprimitive, then $G\left(\chi, 2^{m}\right)=0$. If $2 \nmid c$ and $j \geq J+2$ then, using (18), we can take

$$
\alpha \equiv-c R_{J}^{-1} \equiv-c\left(R_{j}+2^{J-1}\right)^{-1} \equiv-c\left(R_{j}^{-1}-2^{J-1}\right) \bmod 2^{J+1},
$$

and

$$
\chi(\alpha) e_{2^{m}}(\alpha)=\chi\left(-c R_{j}^{-1}\right) e_{2^{m}}\left(-c R_{j}^{-1}\right) \chi\left(1-R_{j} 2^{J-1}\right) e_{2^{m}}\left(c 2^{J-1}\right) .
$$

Checking the four possible $c \bmod 8$,

$$
\frac{1+(-1)^{\lambda} i^{R_{J} c}}{\sqrt{2}}=\frac{1-i^{c}}{\sqrt{2}}=\omega^{-c}\left(\frac{2}{c}\right) .
$$

Now

$$
e_{2^{m}}\left(c 2^{J-1}\right)=e_{2^{m-2}}\left(c 2^{J-3}\right)=\chi\left(5^{2^{J-3}}\right)=\chi\left(1+R_{J-1} 2^{J-1}\right),
$$

where, since $R_{j} \equiv R_{J-1}-2^{J-2} \bmod 2^{J+1}$ and $R_{j} \equiv-1 \bmod 4$,

$$
\begin{aligned}
\left(1-R_{j} 2^{J-1}\right)\left(1+R_{J-1} 2^{J-1}\right) & =1+\left(R_{J-1}-R_{j}\right) 2^{J-1}-R_{j} R_{J-1} 2^{2 J-2} \\
& \equiv 1+2^{2 J-3}+R_{J-1} 2^{2 J-2} \bmod 2^{m}
\end{aligned}
$$


Noting that $R_{s} \equiv-1 \bmod 2^{3}$ for $s \geq 4$ (and checking by hand for $J=3$ or 4 ) gives $1+2 R_{J-1} \equiv$ $R_{2 J-3} \bmod 8$, and

$$
\left(1-R_{j} 2^{J-1}\right)\left(1+R_{J-1} 2^{J-1}\right) \equiv 1+R_{2 J-3} 2^{2 J-3} \bmod 2^{m}
$$

Hence

$$
\chi\left(1-R_{j} 2^{J-1}\right) e_{2^{m}}\left(c 2^{J-1}\right)=\chi\left(5^{2^{2 J-5}}\right)=e_{2^{m-2}}\left(c 2^{2 J-5}\right)= \begin{cases}\omega^{c}, & \text { if } m \text { is even, } \\ \omega^{2 c}, & \text { if } m \text { is odd } .\end{cases}
$$

One can check numerically that the formula still holds for the $2^{m-2}$ primitive mod $2^{m}$ characters when $m=5$. For $m=2,3,4$, one has (21) instead of $2 i \omega, 2^{\frac{3}{2}} \omega^{2}, 2^{2} \chi(c) e_{2^{4}}(c) \omega^{c}$ (so our formula (20) requires an extra factor $\omega^{-1}, \omega^{-1-\chi(-1)}$ or $\chi(-1) \omega^{-2 c}$ respectively).

We shall need the counterpart of (13) for $J_{p^{n}}\left(\chi_{1}, \ldots, \chi_{k}\right)$. We now state a less symmetrical version to allow weaker assumptions on the $\chi_{i}$.

Theorem 2.2. Suppose that $\chi_{1}, \ldots, \chi_{k}$ are mod $p^{m}$ characters with at least one of them primitive and that $m>n$. If $\chi_{1} \cdots \chi_{k}$ is a mod $p^{m-n}$ character, then

$$
J_{p^{n}}\left(\chi_{1}, \ldots, \chi_{k}, p^{m}\right)=p^{-(m-n)} \overline{G\left(\chi_{1} \cdots \chi_{k}, p^{m-n}\right)} \prod_{i=1}^{k} G\left(\chi_{i}, p^{m}\right) .
$$

If $\chi_{1} \cdots \chi_{k}$ is not a mod $p^{m-n}$ character, then $J_{p^{n}}\left(\chi_{1}, \ldots, \chi_{k}, p^{m}\right)=0$.

Recall the well-known properties of Gauss sums (see for example [1, §1.6]),

$$
\left|G\left(\chi, p^{j}\right)\right|= \begin{cases}p^{j / 2}, & \text { if } \chi \text { is primitive } \bmod p^{j}, \\ 1, & \text { if } \chi=\chi_{0} \text { and } j=1, \\ 0, & \text { otherwise. }\end{cases}
$$

So when $\chi_{1} \cdots \chi_{k}$ is a primitive $\bmod p^{m-n}$ character and at least one of the $\chi_{i}$ is a primitive $\bmod p^{m}$ character, we immediately obtain the symmetric form

$$
J_{p^{n}}\left(\chi_{1}, \ldots, \chi_{k}, p^{m}\right)=\frac{\prod_{i=1}^{k} G\left(\chi_{i}, p^{m}\right)}{G\left(\chi_{1} \ldots \chi_{k}, p^{m-n}\right)} .
$$

In particular we recover (13) under the sole assumption that $\chi_{1} \cdots \chi_{k}$ is a primitive mod $p^{m}$ character.

Proof. We first note that if $\chi$ is a primitive character $\bmod p^{j}, j \geq 1$ and $A \in \mathbb{Z}$, then

$$
\sum_{y=1}^{p^{j}} \chi(y) e_{p^{j}}(A y)=\bar{\chi}(A) G\left(\chi, p^{j}\right)
$$


Indeed, for $p \nmid A$ this is plain from $y \mapsto A^{-1} y$. If $p \mid A$ and $j=1$ the sum equals $\sum_{y=1}^{p} \chi(y)=0$. For $j \geq 2$, as $\chi$ is primitive, there exists a $z \equiv 1 \bmod p^{j-1}$ with $\chi(z) \neq 1$. To see this, note that there must be some $a \equiv b \bmod p^{j-1}$ with $\chi(a) \neq \chi(b)$, and we can take $z=a b^{-1}$. So

$$
\sum_{y=1}^{p^{j}} \chi(y) e_{p^{j}}(A y)=\sum_{y=1}^{p^{j}} \chi(z y) e_{p^{j}}(A z y)=\chi(z) \sum_{y=1}^{p^{j}} \chi(y) e_{p^{j}}(A y)
$$

and thus $\sum_{y=1}^{p^{j}} \chi(y) e_{p^{j}}(A y)=0$.

Hence if $\chi_{k}$ is a primitive character $\bmod p^{m}$ we have

$$
\begin{aligned}
& \bar{\chi}_{k}(-1) G\left(\bar{\chi}_{k}, p^{m}\right) \sum_{x_{1}=1}^{p^{m}} \ldots \sum_{x_{k-1}=1}^{p^{m}} \chi_{1}\left(x_{1}\right) \ldots \chi_{k-1}\left(x_{k-1}\right) \chi_{k}\left(p^{n}-x_{1}-\cdots-x_{k-1}\right) \\
& =\bar{\chi}_{k}(-1) \sum_{x_{1}=1}^{p^{m}} \cdots \sum_{x_{k-1}=1}^{p^{m}} \chi_{1}\left(x_{1}\right) \ldots \chi_{k-1}\left(x_{k-1}\right) \sum_{y=1}^{p^{m}} \bar{\chi}_{k}(y) e_{p^{m}}\left(\left(p^{n}-x_{1}-\cdots-x_{k-1}\right) y\right) \\
& =\sum_{y=1}^{p^{m}} \bar{\chi}_{k}(-y) e_{p^{m}}\left(p^{n} y\right)\left(\sum_{x_{1}=1}^{p^{m}} \chi_{1}\left(x_{1}\right) e_{p^{m}}\left(-x_{1} y\right) \cdots \sum_{x_{k-1}=1}^{p^{m}} \chi_{k-1}\left(x_{k-1}\right) e_{p^{m}}\left(-x_{k-1} y\right)\right) \\
& =\sum_{y=1}^{p^{m}} \bar{\chi}_{1 \nmid y} \cdots \chi_{k}(-y) e_{p^{m}}\left(p^{n} y\right)\left(\sum_{x_{1}=1}^{p^{m}} \chi_{1}\left(x_{1}\right) e_{p^{m}}\left(x_{1}\right) \cdots \sum_{x_{k-1}=1}^{p^{m}} \chi_{k-1}\left(x_{k-1}\right) e_{p^{m}}\left(x_{k-1}\right)\right) \\
& =\bar{\chi}_{1 \cdots \chi_{k}(-1) \sum_{y=1}^{p^{m}} \frac{\chi_{1} \cdots \chi_{k}}{\chi_{1}}(y) e_{p^{m}}\left(p^{n} y\right) \prod_{i=1}^{k-1} G\left(\chi_{i}, p^{m}\right) .}
\end{aligned}
$$

If $m>n$ and $\bar{\chi}_{1} \cdots \chi_{k}$ is a mod $p^{m-n}$ character, then

$$
\sum_{\substack{y=1 \\ p \nmid y}}^{p^{m}} \bar{\chi}_{1 \cdots \chi_{k}}(y) e_{p^{m}}\left(p^{n} y\right)=p^{n} \sum_{\substack{y=1 \\ p \nmid y}}^{p^{m-n}} \bar{\chi}_{1 \cdots \chi_{k}}(y) e_{p^{m-n}}(y)=p^{n} G\left(\bar{\chi}_{1} \ldots \chi_{k}, p^{m-n}\right) .
$$

If $\bar{\chi}_{1 \cdots \chi}$ is a primitive character $\bmod p^{j}$ with $m-n<j \leq m$, then by the same reasoning as in (27)

$$
\sum_{\substack{y=1 \\ p \nmid y}}^{p^{m}} \bar{\chi}_{1 \cdots \chi_{k}}(y) e_{p^{m}}\left(p^{n} y\right)=p^{m-j} \sum_{y=1}^{p^{j}} \bar{\chi}_{1 \cdots \chi_{k}}(y) e_{p^{j}}\left(p^{j-(m-n)} y\right)=0
$$

and the result follows from observing that $\overline{G\left(\chi, p^{m}\right)}=\bar{\chi}(-1) G\left(\bar{\chi}, p^{m}\right)$ and, since $\chi_{k}$ is primitive, $\overline{G\left(\chi_{k}, p^{m}\right)}=p^{m} G\left(\chi_{k}, p^{m}\right)^{-1}$. 


\section{Proof of Theorem 1.1}

We assume that $\chi_{1}, \ldots, \chi_{k}$ are all primitive $\bmod p^{m}$ characters and $\chi_{1} \cdots \chi_{k}$ is a primitive $\bmod p^{m-n}$ character, since otherwise from Theorem 2.2 and (25), $J_{p^{n}}\left(\chi_{1}, \ldots, \chi_{k}, p^{m}\right)=0$. In particular we have (26).

We write $R=R_{\left\lceil\frac{m}{2}\right\rceil+2}$, and then by (26) and the evaluation of Gauss sums in Theorem 2.1 we have

$$
\begin{aligned}
J_{p^{n}}\left(\chi_{1}, \ldots, \chi_{k}, p^{m}\right) & =\frac{\prod_{i=1}^{k} G\left(\chi_{i}, p^{m}\right)}{G\left(\chi_{1} \ldots \chi_{k}, p^{m-n}\right)} \\
& =\frac{\prod_{i=1}^{k} p^{m / 2} \chi_{i}\left(-c_{i} R^{-1}\right) e_{p^{m}}\left(-c_{i} R^{-1}\right) \delta_{i}}{p^{(m-n) / 2} \chi_{1} \ldots \chi_{k}\left(-v R^{-1}\right) e_{p^{m-n}\left(-v R^{-1}\right) \delta_{s}}} \\
& =p^{\frac{1}{2}(m(k-1)+n)} \frac{\prod_{i=1}^{k} \chi_{i}\left(c_{i}\right)}{\chi_{1} \ldots \chi_{k}(v)} \delta_{s}^{-1} \prod_{i=1}^{k} \delta_{i},
\end{aligned}
$$

where, as long as $p^{m-n} \neq 27$ and $p^{m} \neq 27$,

$$
\delta_{i}= \begin{cases}\left(\frac{-2 r c_{i}}{p}\right)^{m} \varepsilon_{p^{m}}, & \text { if } p \text { is odd } \\ \left(\frac{2}{c_{i}}\right)^{m} \omega^{c_{i}}, & \text { if } p=2 \text { and } m \geq 5\end{cases}
$$

and

$$
\delta_{s}= \begin{cases}\left(\frac{-2 r v}{p}\right)^{m-n} \varepsilon_{p^{m-n},} & \text { if } p \text { is odd, } \\ \left(\frac{2}{v}\right)^{m-n} \omega^{v}, & \text { if } p=2 \text { and } m-n \geq 5\end{cases}
$$

and the result is plain when $p$ is odd or $p=2, m-n \geq 5$.

For $p^{m-n}=3^{3}, p^{m} \neq 3^{3}$ we get the extra factor $e_{3}(r v)$ from the Gauss sum in the denominator, for $p^{m-n}=p^{m}=3^{3}$ or $p^{m-n} \neq 3^{3}, p^{m}=3^{3}$ the additional factors needed in the Gauss sums cancel. The remaining cases $p=2, m \geq 5$ and $m-n=2,3,4$ follow similarly using the adjustment to $\delta_{s}$ observed at the end of the proof of Theorem 2.1 .

\section{A more direct approach}

We should note that the Cochrane \& Zheng reduction technique in [3] can be applied to directly evaluate the Jacobi sums instead of turning to Gauss sums, via the binomial character sum evaluations of [9] and [10].

CASE A) ODD $p$ AND $m \geq n+2$.

If $b=p^{n} b^{\prime}$ with $p \nmid b^{\prime}$ and $\chi_{2}$ is primitive, then from [9, Theorem 3.1] we have

$$
J_{b}\left(\chi_{1}, \chi_{2}, p^{m}\right)=\sum_{x=1}^{p^{m}} \chi_{1}(x) \chi_{2}(b-x)=\sum_{x=1}^{p^{m}} \overline{\chi_{1} \chi_{2}}(x) \chi_{2}(b x-1)
$$




$$
=p^{\frac{m+n}{2}} \overline{\chi_{1} \chi_{2}}\left(x_{0}\right) \chi_{2}\left(b x_{0}-1\right)\left(\frac{-2 c_{2} r b^{\prime} x_{0}}{p}\right)^{m-n} \varepsilon_{p^{m-n}}
$$

with an extra factor $e_{3}\left(r\left(c_{1}+c_{2}\right) / p^{n}\right)$ needed when $p^{m-n}=27, n>0$, where $x_{0}$ is a solution to the characteristic equation

$$
c_{1}+c_{2}-c_{1} b x \equiv 0 \bmod p^{\left\lfloor\frac{m+n}{2}\right\rfloor+1}, \quad p \nmid x(b x-1) .
$$

If (29) has no solution $\bmod p^{\left\lfloor\frac{m+n}{2}\right\rfloor}$, then $J_{b}\left(\chi_{1}, \chi_{2}, p^{m}\right)=0$. In particular we see the following.

(i) If $p \mid c_{1}$ and $p \nmid c_{2}$, then $J_{b}\left(\chi_{1}, \chi_{2}, p^{m}\right)=0$.

(ii) If $p \nmid c_{1} c_{2}\left(c_{1}+c_{2}\right)$ then

$$
J_{b}\left(\chi_{1}, \chi_{2}, p^{m}\right)=p^{\frac{m}{2}} \chi_{1} \chi_{2}(b) \chi_{1}\left(c_{1}\right) \chi_{2}\left(c_{2}\right) \overline{\chi_{1} \chi_{2}}\left(c_{1}+c_{2}\right) \delta_{2}
$$

where

$$
\delta_{2}=\left(\frac{-2 r}{p}\right)^{m}\left(\frac{c_{1} c_{2}\left(c_{1}+c_{2}\right)}{p}\right)^{m} \varepsilon_{p^{m}}
$$

(iii) If $p \nmid c_{1}$ and $b=p^{n} b^{\prime}, p \nmid b^{\prime}$ with $n<m-1$ then $J_{b}\left(\chi_{1}, \chi_{2}, p^{m}\right)=0$ unless $p^{n} \|\left(c_{1}+c_{2}\right)$ in which case writing $w=\left(c_{1}+c_{2}\right) / p^{n}$, we get

$$
J_{b}\left(\chi_{1}, \chi_{2}, p^{m}\right)=p^{\frac{m+n}{2}} \chi_{1} \chi_{2}\left(b^{\prime}\right) \frac{\chi_{1}\left(c_{1}\right) \chi_{2}\left(c_{2}\right)}{\chi_{1} \chi_{2}(w)}\left(\frac{-2 r}{p}\right)^{m-n}\left(\frac{c_{1} c_{2} w}{p}\right)^{m-n} \varepsilon_{p^{m-n}}
$$

with an extra factor $e_{3}(r w)$ needed when $p^{m-n}=27, n>0$.

To see (ii) observe that if $p \mid b$, then $J_{b}\left(\chi_{1}, \chi_{2}, p^{m}\right)=0$, and if $p \nmid b$, then we can take $x_{0} \equiv\left(c_{1}+c_{2}\right) c_{1}^{-1} b^{-1} \bmod p^{m}$ (and hence $b x_{0}-1=c_{2} c_{1}^{-1}$ ). Similarly for (iii) if $p^{n} \|\left(c_{1}+c_{2}\right)$ we can take $x_{0} \equiv p^{-n}\left(c_{1}+c_{2}\right) c_{1}^{-1}\left(b^{\prime}\right)^{-1} \bmod p^{m}$.

Of course we can write the generalized sum in the form

$$
\begin{aligned}
J_{p^{n}}\left(\chi_{1}, \ldots, \chi_{k}, p^{m}\right) & =\sum_{x_{3}=1}^{p^{m}} \ldots \sum_{x_{k}=1}^{p^{m}} \chi_{3}\left(x_{3}\right) \ldots \chi_{k}\left(x_{k}\right) \sum_{\substack{x_{1}=1 \\
b:=p^{n}-x_{3}-\cdots-x_{k}}}^{p^{m}} \chi_{1}\left(x_{1}\right) \chi_{2}\left(b-x_{1}\right) \\
& =\sum_{x_{3}=1}^{p^{m}} \cdots \sum_{x_{k}=1}^{p^{m}} \chi_{3}\left(x_{3}\right) \ldots \chi_{k}\left(x_{k}\right) J_{b}\left(\chi_{1}, \chi_{2}, p^{m}\right),
\end{aligned}
$$

Hence assuming that at least one of the $\chi_{i}$ is primitive $\bmod p^{m}$ (and reordering the characters as necessary) we see from (i) that $J_{p^{n}}\left(\chi_{1}, \ldots, \chi_{k}, p^{m}\right)=0$ unless all the characters are primitive $\bmod p^{m}$. Also when $k=2, \chi_{1}, \chi_{2}$ primitive, we see from (iii) that $J_{p^{n}}\left(\chi_{1}, \chi_{2}, p^{m}\right)=0$ unless $\chi_{1} \chi_{2}$ is induced by a primitive $\bmod p^{m-n}$ character, in which case we recover the formula 
in Theorem 1.1 on observing that $\left(\frac{c_{1} c_{2}}{p}\right)^{n} \varepsilon_{p^{m-n}}^{2}=\varepsilon_{p^{m}}^{2}$; this is plain when $n$ is even, for $n$ odd observe that $\left(\frac{c_{1} c_{2}}{p}\right)=\left(\frac{\left(c_{1}+c_{2}\right)^{2}-\left(c_{1}-c_{2}\right)^{2}}{p}\right)=\left(\frac{-1}{p}\right)$.

We show that a simple induction recovers the formula for all $k \geq 3$. We assume that all the $\chi_{i}$ are primitive mod $p^{m}$ and observe that when $k \geq 3$ we can further assume (reordering as necessary) that $\chi_{1} \chi_{2}$ is also primitive $\bmod p^{m}$, since if $\chi_{1} \chi_{3}, \chi_{2} \chi_{3}$ are not primitive then $p \mid\left(c_{1}+c_{3}\right)$ and $p \mid\left(c_{2}+c_{3}\right)$ and $\left(c_{1}+c_{2}\right) \equiv-2 c_{3} \not \equiv 0 \bmod p$ and $\chi_{1} \chi_{2}$ is primitive. Hence from (ii) we can write

$$
\begin{aligned}
J_{p^{m}}\left(\chi_{1}, \ldots, \chi_{k}, p^{m}\right) & =\frac{\chi_{1}\left(c_{1}\right) \chi_{2}\left(c_{2}\right)}{\chi_{1} \chi_{2}\left(c_{1}+c_{2}\right)} p^{\frac{m}{2}} \delta_{2} \sum_{\chi_{3}=1}^{p^{m}} \ldots \sum_{x_{k}=1}^{p^{m}} \chi_{3}\left(x_{3}\right) \ldots \chi_{k}\left(x_{k}\right) \chi_{1} \chi_{2}(b) \\
& =p^{\frac{m}{2}} \chi_{1}\left(c_{1}\right) \chi_{2}\left(c_{2}\right) \overline{\chi_{1} \chi_{2}}\left(c_{1}+c_{2}\right) \delta_{2} J_{p^{n}}\left(\chi_{1} \chi_{2}, \chi_{3}, \ldots, \chi_{k}, p^{m}\right) .
\end{aligned}
$$

Assuming the result for $k-1$ characters we have $J_{p^{n}}\left(\chi_{1} \chi_{2}, \chi_{3}, \ldots, \chi_{k}, p^{m}\right)=0$ unless $\chi_{1} \cdots \chi_{k}$ is induced by a primitive $\bmod p^{m-n}$ character, in which case

$$
J_{p^{n}}\left(\chi_{1} \chi_{2}, \chi_{3}, \ldots, \chi_{k}, p^{m}\right)=p^{\frac{m(k-2)+n}{2}} \chi_{1} \chi_{2}\left(c_{1}+c_{2}\right) \delta_{3} \prod_{i=3}^{k} \chi_{i}\left(c_{i}\right) \overline{\chi_{1} \ldots \chi_{k}}(v)
$$

where

$$
\delta_{3}=\left(\frac{-2 r}{p}\right)^{m(k-2)+n}\left(\frac{v}{p}\right)^{m-n}\left(\frac{\left(c_{1}+c_{2}\right) c_{3} \ldots c_{k}}{p}\right)^{m} \varepsilon_{p^{m}}^{k-1} \varepsilon_{p^{m-n}}^{-1},
$$

plus an additional factor $e_{3}(r v)$ if $p^{m-n}=27, n>0$. Our formula for $k$ characters then follows on observing that $\delta_{2} \delta_{3}=\delta$.

CASE B) WHEN $p=2$ AND $m \geq n+5$.

Suppose that $\chi_{2}$ is primitive $\bmod 2^{m}$, that is $2 \nmid c_{2}$, and $b=2^{n} b^{\prime}$ with $2 \nmid b^{\prime}$ and $m \geq n+5$. In this case from [10, Theorem 1.1] we similarly have $J_{b}\left(\chi_{1}, \chi_{2}\right)=0$ unless $2 \nmid c_{1}$ and $2^{n} \| c_{1}+c_{2}$, in which case

$$
J_{b}\left(\chi_{1}, \chi_{2}, 2^{m}\right)=2^{\frac{1}{2}(m+n)} \overline{\chi_{1} \chi_{2}}\left(x_{0}\right) \chi_{2}\left(b x_{0}-1\right) \begin{cases}1, & \text { if } m-n \text { is even, } \\ \omega^{h}\left(\frac{2}{h}\right), & \text { if } m-n \text { odd }\end{cases}
$$

where $x_{0}$ is a solution to

$$
-\left(c_{1}+c_{2}\right)\left(b x_{0}-1\right)+c_{2} b x_{0} R_{N} R_{N+n}^{-1} \equiv 0 \bmod 2^{N+n+3},
$$

with $2 \nmid x_{0}\left(b x_{0}-1\right)$ and

$$
\omega:=e_{8}(1), \quad N:=\left\lceil\frac{1}{2}(m-n)\right\rceil \geq 3, v:=\frac{c_{1}+c_{2}}{2^{n}}, h: \equiv-\left(2^{n}-1\right) v \bmod 8 .
$$


From the relations (17) we obtain

$$
R_{l+n} R_{l}^{-1}-1=2^{l-1} \mu_{l}, \mu_{l} \equiv\left(2^{n}-1\right) R_{l} \bmod 8
$$

where $R_{2}=1, R_{3}=3$, and $R_{j} \equiv-1 \bmod 8$ for $j \geq 4$. Hence, taking $x_{0}=v b^{-1}\left(c_{1}+c_{2}-c_{2} R_{N} R_{N+n}^{-1}\right)^{-1}$, we get

$$
J_{b}\left(\chi_{1}, \chi_{2}, 2^{m}\right)=2^{\frac{1}{2}(m+n)} \chi_{1} \chi_{2}\left(b^{\prime}\right) \frac{\chi_{1}\left(c_{1}\right) \chi_{2}\left(c_{2}\right)}{\chi_{1} \chi_{2}(v)}\left(\frac{2}{v}\right)^{m-n} \epsilon
$$

with

$$
\epsilon:=\overline{\chi_{1} \chi_{2}}\left(1+2^{N-1} \mu_{N}\right) \chi_{1}\left(1+c_{1}^{-1} v \mu_{N} 2^{N+n-1}\right) \begin{cases}1, & \text { if } m-n \text { is even } \\ \omega^{-\left(2^{n}-1\right) v}\left(\frac{2}{2^{n}-1}\right), & \text { if } m-n \text { is odd }\end{cases}
$$

where $\overline{\chi_{1} \chi_{2}}$ is a primitive mod $2^{m-n}$ character. Expanding binomially, observing that $2(N+$ $n-1) \geq m$ if $n \geq 2$ or $m$ is even, and $2(N+n-1)=m-1$ if $n=1$ and $m$ is odd, one readily obtains

$$
1+c_{1}^{-1} v \mu_{N} 2^{N+n-1} \equiv\left(1+R_{N+n-1} 2^{N+n-1}\right)^{\kappa}=5^{2^{N+n-3} \kappa} \bmod 2^{m},
$$

with

$$
\kappa:=c_{1}^{-1} v \mu_{N} R_{N+n-1}^{-1}+ \begin{cases}\frac{1}{2}\left(v-c_{1}\right) 2^{(m-1) / 2}, & \text { if } n=1, m \text { odd } \\ 0, & \text { else. }\end{cases}
$$

Similarly,

$$
\begin{aligned}
1+2^{N-1} \mu_{N} & \equiv 1+R_{N-1} 2^{N-1} \mu_{N} R_{N-1}^{-1} \equiv 1+2^{N-1} R_{N-1} \mu_{N} R_{N+n-1}^{-1}\left(1+2^{N-2} \mu_{N-1}\right) \\
& \equiv 1+R_{N-1} 2^{N-1}\left(\mu_{N} R_{N+n-1}^{-1}+2^{N-2} R_{N} R_{N-1} R_{N+n-1}^{-1}\right) \bmod 2^{m-n}
\end{aligned}
$$

and, since $3(N-1) \geq m-n$,

$$
1+2^{N-1} \mu_{N} \equiv\left(1+R_{N-1} 2^{N-1}\right)^{\mu_{N} R_{N+n-1}^{-1}-2^{N-2}\left(2^{n}-1\right)}=5^{2^{N-3}\left(\mu_{N} R_{N+n-1}^{-1}-2^{N-2}\left(2^{n}-1\right)\right)} \bmod 2^{m-n} .
$$

Hence, checking the possibilities $\bmod 8$, recalling that $2^{n} \| c_{1}+c_{2}$,

$$
\begin{aligned}
\epsilon & =e_{2^{m-n-2 N+3}}\left(\left(2^{n}-1\right) v\right) \cdot \begin{cases}(-1)^{\frac{1}{2}\left(\nu-c_{1}\right)}, & \text { if } m-n \text { is even and } n=1, \\
1, & \text { if } m-n \text { is even and } n \geq 2, \\
\omega^{-\left(2^{n}-1\right) \nu}\left(\frac{2}{2^{n}-1}\right), & \text { if } m-n \text { odd. }\end{cases} \\
& =\omega^{\left(2^{n}-1\right) v}\left(\frac{2}{c_{1} c_{2}}\right)^{m}
\end{aligned}
$$

and we obtain the $p=2, k=2$ result of Theorem 1.1. As in the case of odd $p$ we can deduce from the $k=2$ result that $J_{b}\left(\chi_{1}, \ldots, \chi_{k}, 2^{m}\right)=0$ if the sum contains both primitive and 
imprimitive $\chi_{i} \bmod 2^{m}$. Hence in the following we assume that all the $\chi_{i}$ are primitive mod $2^{m}$.

For $k=3$ we observe from parity considerations that $J_{b}\left(\chi_{1}, \chi_{2}, \chi_{3}, 2^{m}\right)=0$ if $b$ is even, while if $b$ is odd we can make the change of variables $x_{i} \mapsto b x_{i}$. Hence in either case

$$
J_{b}\left(\chi_{1}, \chi_{2}, \chi_{3}, 2^{m}\right)=\chi_{1} \chi_{2} \chi_{3}(b) J\left(\chi_{1}, \chi_{2}, \chi_{3}, 2^{m}\right) .
$$

Now at least one of $\chi_{1} \chi_{2}, \chi_{1} \chi_{3}, \chi_{2} \chi_{3}$ is primitive $\bmod 2^{m-1}$ (since they are all mod $2^{m-1}$ characters and $\chi_{1}^{2}=\chi_{1} \chi_{2} \cdot \chi_{1} \chi_{3} \cdot \overline{\chi_{2} \chi_{3}}$ is primitive $\bmod 2^{m-1}$ ). We suppose that $\chi_{1} \chi_{2}$ is primitive $\bmod 2^{m-1}$, i.e. $2 \| c_{1}+c_{2}$. Then

$$
\begin{aligned}
J\left(\chi_{1}, \chi_{2}, \chi_{3}, 2^{m}\right) & =\sum_{\substack{x_{3}=1 \\
x_{3} \text { odd }}}^{2^{m}} \chi_{3}\left(x_{3}\right) J_{1-x_{3}}\left(\chi_{1}, \chi_{2}, 2^{m}\right) \\
& =2^{\frac{1}{2}(m+1)} \frac{\chi_{1}\left(c_{1}\right) \chi_{2}\left(c_{2}\right)}{\chi_{1} \chi_{2}\left(\frac{c_{1}+c_{2}}{2}\right)}\left(\frac{2}{\frac{c_{1}+c_{2}}{2}}\right)^{m-1}\left(\frac{2}{c_{1} c_{2}}\right)^{m} \omega^{\frac{1}{2}\left(c_{1}+c_{2}\right)} \sum_{\substack{x_{3}=1 \\
x_{3} \text { odd }}}^{2^{m}} \chi_{3}\left(x_{3}\right) \chi_{1} \chi_{2}\left(\frac{1-x_{3}}{2}\right) .
\end{aligned}
$$

Now

$$
\left.\sum_{\substack{x_{3}=1 \\ x_{3} \text { odd }}}^{2^{m}} \chi_{3}\left(x_{3}\right) \chi_{1} \chi_{2}\left(\frac{1-x_{3}}{2}\right)=\frac{1}{2} \sum_{\substack{x_{3}=1 \\ 1-x_{3} \equiv 2 x \bmod 2^{m}}}^{2^{m}} \sum_{\substack{x=1 \\ 2^{m}}} x_{3}\right) \chi_{1} \chi_{2}(x)
$$

which, from the change of variables $x \mapsto x^{-1}, x_{3} \mapsto-x_{3} x^{-1}$ and the $k=2$ result, equals

$$
\begin{aligned}
& \frac{1}{2} \chi_{3}(-1) \quad \sum_{\begin{array}{c}
x_{3}=1 \\
x+x_{3} \equiv 2 \bmod 2^{m}
\end{array}}^{2^{m}} \sum_{3=1}^{2^{m}}\left(x_{3}\right) \overline{\chi_{1} \chi_{2} \chi_{3}}(x)= \\
& 2^{\frac{1}{2}(m-1)} \chi_{3}(-1) \frac{\overline{\chi_{1} \chi_{2} \chi_{3}}\left(-\left(c_{1}+c_{2}+c_{3}\right)\right) \chi_{3}\left(c_{3}\right)}{\overline{\chi_{1} \chi_{2}}\left(-\frac{1}{2}\left(c_{1}+c_{2}\right)\right)}\left(\frac{2}{-\frac{c_{1}+c_{2}}{2}}\right)^{m-1}\left(\frac{2}{-\left(c_{1}+c_{2}+c_{3}\right) c_{3}}\right)^{m} \omega^{-\frac{1}{2}\left(c_{1}+c_{2}\right)},
\end{aligned}
$$

since $\chi_{3} \overline{\chi_{1} \chi_{2} \chi_{3}}=\overline{\chi_{1} \chi_{2}}$ and $2 \nmid c_{i}$ ensures that $2 \nmid c_{1}+c_{2}+c_{3}$. Hence

$$
J\left(\chi_{1}, \chi_{2}, \chi_{3}, 2^{m}\right)=2^{m} \frac{\chi_{1}\left(c_{1}\right) \chi_{2}\left(c_{2}\right) \chi_{3}\left(c_{3}\right)}{\chi_{1} \chi_{2} \chi_{3}\left(c_{1}+c_{2}+c_{3}\right)}\left(\frac{2}{c_{1}+c_{2}+c_{3}}\right)^{m}\left(\frac{2}{c_{1} c_{2} c_{3}}\right)^{m},
$$

and we recover Theorem 1.1 when $k=3$ (note $J_{p^{n}}\left(\chi_{1}, \chi_{2}, \chi_{3}, 2^{m}\right)=0$ unless $n=0$ ).

For $k \geq 4$ we use (30) to write

$$
J_{b}\left(\chi_{1}, \ldots, \chi_{k}, 2^{m}\right)=J_{b}\left(\chi_{1} \chi_{2} \chi_{3}, \chi_{4}, \ldots, \chi_{n}, 2^{m}\right) J\left(\chi_{1}, \chi_{2}, \chi_{3}, 2^{m}\right)
$$

and the Theorem 1.1 result for general $k$ follows easily by induction. 


\section{References}

[1] B.C. Berndt, R.J. Evans and K. S. Williams, Gauss and Jacobi Sums, Canadian Math. Soc. series of monographs and advanced texts, vol. 21, Wiley, New York 1998.

[2] T. Cochrane, Exponential sums modulo prime powers, Acta Arith., 101 (2002), no. 2, 131-149.

[3] T. Cochrane and Z. Zheng, Pure and mixed exponential sums, Acta Arith., 91 (1999), no. 3, 249-278 (for errata see http:// www.math.ksu.edu/ cochrane/research/research09.html).

[4] T. Cochrane and Z. Zheng, A survey on pure and mixed exponential sums modulo prime powers, Number theory for the millennium, I (Urbana, IL, 2000), 273-300, A K Peters, Natick,MA, 2002.

[5] R. Lidl and H. Niederreiter, Finite Fields, Encyclopedia of Mathematics and its applications 20, 2nd edition, Cambridge University Press, 1997.

[6] J.-L. Mauclaire, Sommes de Gauss modulo $p^{\alpha}$, I \& II, Proc. Japan Acad. Ser. A, 59 (1983), 109-112 \& 161-163.

[7] R. Odoni, On Gauss sums $\left(\bmod p^{n}\right), n \geq 2$, Bull. London Math. Soc., 5 (1973), 325-327.

[8] V. Pigno and C. Pinner, Twisted monomial Gauss sums modulo prime powers, Func. Approx. Comment. Math., 51 (2014), no. 2, 285-301.

[9] V. Pigno and C. Pinner, Binomial character sums modulo prime powers, J. Théor. Nombres Bordeaux, 28 (2016), no. 1, 39-53.

[10] V. Pigno, C. Pinner and J. Sheppard, Evaluating binomial character sums modulo powers of two, J. Math. Res. Appl., 35 (2015), no. 2, 137-142.

[11] J. Wang, On the Jacobi sums $\bmod P^{n}$, J. Number Theory, 39 (1991), 50-64.

[12] W. Zhang and W. Yao, A note on the Dirichlet characters of polynomials, Acta Arith., 115 (2004), no. 3, 225-229.

[13] W. Zhang and Z. Xu, On the Dirichlet characters of polynomials in several variables, Acta Arith., 121 (2006), no. 2, 117-124.

Department of Mathematics, University of Southern Indiana, Evansville, IN 47712.

E-mail: m.ostergaard@usi.edu

Department of Mathematics \& Statistics, California State University, Sacramento, Sacramento, CA 95819.

E-mail: vincent.pigno@csus.edu

Department of Mathematics, Kansas State University, Manhattan, KS 66506.

E-mail: pinner@math.ksu.edu 